\title{
Las transformaciones del estado y del derecho administrativo en el marco de la integración y la globalización
}

\author{
AUGUSTO RAMÓN CHÁVEZ MARÍN*
}

\section{RESUMEN}

Al reflexionar sobre las transformaciones contemporáneas del Derecho Administrativo, este trabajo examina en un primer espacio algunas consecuencias de los procesos de integración comunitaria sobre el Derecho Interno y, específicamente, sobre la estructura del ordenamiento jurídico nacional generada por los fenómenos de delegación de soberanía a favor de autoridades supranacionales. Siguiendo la misma línea de análisis, en un segundo aparte el autor estudia la atribución de funciones regulatorias a las denominadas agencias de regulación, ya por el legislador interno o por la autoridad comunitaria, bien de naturaleza legislativa o bien administrativa, avizorando sus consecuencias. Por último, revisa en forma general el amplio horizonte de la redefinición del modelo de Estado requerida e impuesta por las nuevas circunstancias marcadas por fenómenos como la integración, la mundialización, la desregulación y las corrientes privatizadoras que conllevan nuevas definiciones del Derecho Administrativo.

\section{PALABRAS CLAVE}

Delegación de soberanía, leyes comunitarias, agencias de regulación, nuevo modelo de Estado, redefinición del Derecho Administrativo.

\begin{abstract}
Reflecting about the contemporary transformations of the Administrative Law, on a first stage this work examinantes some results of the community's integration processes about the Intern Law, and specifically, about the National Set of Laws generated by the Sovereignty's Delegation phenomena on
\end{abstract}

\footnotetext{
Docente de la Facultad de Derecho de la Universidad Santo Tomás
} 
favor of supranational authorities. On a second stage, following the same analysis line, the author studies the attribution of regulatory functions to the named regulation Agencies, either done by internal legislator or the community's authority, those attributions could be of a legislative or administrative nature. The investigator also forsees the results of this attributions. Finally, it reviews on a general way the broad horizon of the State's Model's redefinition imposed and required by the new circumstances marked by phenomena as the integration, the globalization, the release of regulations and the privatization streams that carry along new definitions of the Administrative Law.

\section{KEY WORDS}

Sovereignty's Delegation, Community's Laws, Regulation Agencies, New Definitions of the Administrative Law.

\section{INTRODUCCIÓN}

Múltiples son las reflexiones que suscitan las consecuencias de los procesos de integración y globalización sobre el Derecho en general y, particularmente, sobre el Derecho Administrativo. Sin embargo, en este trabajo nos referiremos solamente a algunos aspectos de tales efectos: los que tienen que ver con la distribución de competencias en la reglamentación de ciertos ámbitos de la vida social entre las autoridades multilaterales y las nacionales, así como a las nuevas corrientes privatizadoras en la gestión de los asuntos del Estado. Haremos, en tercer lugar, algunos comentarios frente a las Agencias de Regulación: sujeto administrativo de naturaleza especial, llamado a desempeñar un importante papel en el horizonte del Derecho Administrativo. Dentro del amplio espectro de las discusiones que genera la integración y la globalización frente a la concepción del Estado y del Derecho Administrativo, el examen de estos temas presenta un especial interés.

\section{LA INTEGRACIÓN Y LA ESTRUCTURA DEL ORDENAMIENTO JURÍDICO NACIONAL}

Un aspecto sobre el que ha operado una transformación del Derecho Administrativo a causa del fenómeno integracionista tiene que ver con la estructura del Ordenamiento Jurídico Nacional de los estados miembros de ciertos acuerdos multilaterales. Ello ocurre, específicamente, con la proyección de los efectos en los derechos nacionales de los Estados que son partes en el Acuerdo de Cartagena.

El tema guarda relación y tiene su fuente en un elemento propio del Derecho Constitucional que se proyecta sobre el Derecho Administrativo, constituido por el concepto de soberanía. Como se recuerda, ésta ha sido entendida contemporáneamente como un atributo del Estado, cuyo depositario primario es la nación o el pueblo, tal cual lo han contemplado las Constituciones Colombianas de 1886 y 1991. Pero, se ha considerado especialmente que esa condición del concepto mismo de Estado se ejerce de una manera suprema y autónoma frente a 
otros poderes internos o externos ${ }^{1}$ e implica la competencia exclusiva para crear el Derecho ${ }^{2}$.

Ahora bien, como lo afirma el Consejo de Estado refiriéndose a la Junta del Acuerdo de Cartagena, el ordenamiento jurídico comunitario se configura como "un conjunto de normas cuyos sujetos activos y pasivos son los estados miembros y sus ciudadanos, dotado de órganos propios con poderes soberanos en ciertas materias específicas y en donde sus miembros han cedido parcialmente la soberanía nacional en los ámbitos que se han reservado a la autoridad comunitaria" $^{3}$. Conforme a ello, la soberanía no es un atributo exclusivo de los Estados sino que también la tienen los órganos multilaterales originados en acuerdos internacionales, nacida en la delegación que hacen los Estados suscriptores para ciertas materias específicas. Se presenta así una "soberanía compartida" entre estos dos niveles de organizaciones, el internacional y el nacional.

Pero, lo más interesante del tema hace relación a los poderes específicos que emanan de esa cesión de soberanía en cuanto a la creación del Derecho: a tales órganos comunitarios se atribuye la expedición de normas de carácter supranacional, de aplicación inmediata y directa en el orden jurídico interno de cada Estado miembro de la comunidad, en las materias reservadas a su competencia en los convenios correspondientes. Así ocurre, específicamente, en respecto a la regulación de la propiedad industrial, pa- tentes y marcas, cuya expedición, atribuida por la Constitución de 1991 al Congreso de la República (art. 150-24), ha sido transferida al legislador subregional, como lo ha precisado el Consejo de Estado en el fallo citado. En tales materias, sin embargo, a los países miembros del pacto internacional se atribuyen competencias residuales o complementarias de naturaleza regulatoria, las cuales se deben ejercer de acuerdo con la política legislativa común e igualmente con la distribución de funciones propia de los ordenamientos constitucionales internos.

Y esas competencias residuales o complementarias generan dos tipos de disposiciones en lo que respecta al Derecho colombiano: las normas de jerarquía legal y las de naturaleza administrativa. Las primeras, cuya expedición se atribuye al Congreso de la República, tienen por objeto Ilenar los vacíos no regulados por las normas comunitarias. Las últimas tienen por fin asegurar el cumplimiento de las normas comunitarias; en tal virtud, de reglamentar éstas, y su expedición se atribuye al Presidente de la República en ejercicio de la potestad reglamentaria establecida por el art. 189 - 11 de la Carta. En la sentencia ya citada, el Consejo de Estado reitera el criterio expuesto en otras oportunidades, indicando que esta potestad se ejerce por el Presidente frente a las normas comunitarias, dada la posición sustitutiva de las leyes nacionales que ocupan tales disposiciones, esto es, su fuerza normativa de ley incorporada al ordenamiento nacional ${ }^{4}$.

\footnotetext{
Véase VIDAL PERDOMO, Jaime. Derecho Constitucional General e Instituciones Políticas Colombianas, Bogotá: Légis, 1998, p. 66.

2 PALACIOS MEjía Hugo. Introducción a la Teoría del Estado. Bogotá: Témis, $2^{a}$ ed.

3 Sala de lo C.A., Sección 1a ${ }^{\text {. }}$, M.P. Manuel Urueta Ayola, Sentencia del 27 de enero del 2000, Exp. 4630. En este asunto el Consejo de Estado decidió sobre la legalidad del Decreto 698 de 1997, por el cual se reglamenta el art. 107 de la Decisión 344 de 1993 expedida por la Comisión del Acuerdo de Cartagena. El decreto demandado fue dictado, especialmente, en ejercicio de facultades otorgadas por los art. 143 y 144 de ese acto comunitario, sobre cuyo alcance versa en gran medida el fallo de nuestro Contencioso Administrativo.

4 Véanse las sentencias de la Sección 1a. De la Sala de lo Contencioso Administrativo del 28 de febrero de 1991, exp. 836, M.P. Libardo Rodríguez y de 10 de noviembre de 1992, exp. 2009, Ernesto Rafael Ariza.
} 
Para el autor de estas líneas, la categorización de las normas expuestas, derivada del ejercicio de una soberanía parcialmente cedida a los órganos supranacionales, tiene un carácter profundamente modificatorio del alcance de importantes elementos de la teoría de la estructura del orden jurídico interno, que se proyecta sobre el ámbito de las facultades propias de dos de los órganos del Estado creadores del Derecho, como son el Legislador y el Ejecutivo. La norma supranacional sustituye con su imperio la ley nacional y la potestad reglamentaria se ejerce no sobre las leyes emanadas del Legislador nacional, sino sobre las "leyes comunitarias". Esta situación genera una compleja delimitación de competencias entre esas tres órbitas (órganos supranacional, legislativo y ejecutivo), cuyas implicaciones deberán ser estudiadas por el nuevo Derecho Administrativo ${ }^{5}$.

\section{LA ATRIBUCIÓN DE FUNCIONES A LAS AGENCIAS DE REGULACIÓN}

Adicionalmente, en torno a este tema, una situación especial puede presentarse cuando directamente la Constitución Política, la misma ley o el propio Presidente de la República mediante delegación, han asignado a un órgano autónomo de orden administrativo o bien a instituciones de carácter particular, la facultad regulatoria sobre ciertos aspectos de la vida social. Pero si ello queda librado al Derecho interno de cada país, de otra parte esa puede ser una tendencia en el campo comunitario, cuando quiera que las mismas normas de esa índole (comunitarias), con el carácter imperativo y supremo que les es pro- pio, asignen directamente la competencia para regular ciertas materias a las denominadas Web Regulatory o agencias de regulación. Estas instituciones constituyen organismos autónomos frente al Legislador ordinario y guardan relativa independencia en relación con el Ejecutivo. De tal manera se produce un desplazamiento por parte de estas agencias regulatorias respecto del Legislador, arrogándose el poder de regular, con fuerza vinculante semejante a la ley, las materias a ellas atribuidas por el Derecho Comunitario. Si lo anterior ocurre desde el punto de vista de la autorización para la expedición de normas regulatorias, las cuales, podría afirmarse, ocupan el mismo espacio y jerarquía de la ley, lo propio puede ocurrir respecto a la aplicación de las normas comunitarias, esto es, al desarrollo de las actividades necesarias para garantizar el cumplimiento de las disposiciones emanadas de los órganos multilaterales. En el ejercicio de esta función de orden ejecutivo o administrativo, y ya no legislativo como la anterior, dichas agencias desplazan al órgano ejecutivo del Estado Nacional.

Los fenómenos revisados en éste y el punto anterior pueden significar un efecto colateral de orden político, constitucional y administrativo, consistente en la pérdida de importancia del órgano colegiado de representación popular, en su condición de autoridad legisladora, en tanto se produce una importante reducción del ámbito de sus competencias regulatorias. Así, podría verse reducido el papel del Congreso de la República a su función de constituyente derivado y de control político, operando la transferencia

5 Sobre este punto, por ejemplo, el Tribunal de Justicia de la Comunidad Andina, en la interpretación 28-IP-99, mencionada por la citada Sentencia del 27 de enero del 2000, exp. 4630, indica que "El artículo 144 de la Decisión 344 de la Comisión del Acuerdo de Cartagena está inspirado en los principios fundamentales de aplicación directa y preferente de la ley comunitaria, que supone que el ordenamiento comunitario opera sin necesidad de complemento legislativo interno y con preeminencia sobre la ley nacional, siendo inaplicables las disposiciones de derecho interno que contradigan o sean irreconciliables con la norma andina" (negrilla fuera de texto). Igualmente dicho Tribunal hizo referencia a que no es posible expedir normas sobre el mismo asunto que versa el ordenamiento comunitario, salvo que sean necesarias para la correcta aplicación de aquellas, por lo cual para que tenga validez la legislación interna se requiere que verse sobre asuntos no regulados en absoluto por la Comunidad. 
de sus funciones legislativas a dichas agencias, Ilamadas a expedir regulaciones que se adapten a los cambios permanentes de las condiciones económicas, sociales y culturales del mundo actual. Esta parece ser la situación que se vislumbra en Colombia, por ejemplo, en el campo de las comisiones de regulación en materia de servicios públicos domiciliarios y podría replicarse en otros campos.

\section{EL EJERCICIO DE FUNCIONES ADMINIS- TRATIVAS POR PARTICULARES}

También se ve desde la óptica de los fenómenos expuestos cómo el ejercicio de la función administrativa y el protagonismo de órganos ubicados en el marco de la Rama ejecutiva van pasando a un primer plano, frente al papel tradicionalmente principal del Legislador. Pero si ello ocurre en el campo de la integración, de otra parte y simultáneamente las actuales condiciones del mundo globalizado dan prioridad a la Administración del Estado, exigiendo y esperando del gobierno la facilitación de una infraestructura institucional que desde lo público promueva y garantice, especialmente, el libre desarrollo del mercado, el ejercicio de la propiedad, la posibilidad de contratar autónomamente, el acceso igual a la información y la igualdad en la competencia.

Se indica la necesidad, al lado de las reformas del marco regulatorio apropiadas a los fines que se acaban de señalar, de una adecuada gestión del gobierno, la cual propenda por una redefinición del Estado, que opere enfáticamente en la Administración: respecto a la gestión económica y fiscal debe impedir los altos índices de inflación, el gasto improductivo, los desequilibrios fiscales y el déficit en la balanza de pagos; mientras que, de otra parte, pero igualmente orbitando en lo administrativo, se propone la reducción del sector público que afirme el retiro del Estado de actividades comerciales que pueden ser realizadas por particulares, así como la privatización de las empresas públicas ineficientes y la prestación de su parte de los servicios públicos esenciales. Todo lo cual implica la necesidad colateral de redefinir el papel del Derecho Administrativo, adecuándolo a las nuevas condiciones.

Queremos hacer énfasis en el hecho de que en el marco anterior se plantea, anexo a los temas de la privatización, pero a nuestro parecer con un alcance que debe entenderse como distinto, la participación de los particulares en la gestión de los servicios públicos y, en general, de las funciones públicas y administrativas. Respecto a ello es interesante observar como en la Carta de 1991 se propone un nuevo modelo de Estado, el cual prevé en forma genérica esa posibilidad, en cuyo sentido se destacan los artículos. $26,123,210,270,365,48,49,67$ y 68 . Por su parte, normas de jerarquía legal han venido atribuyendo, aún antes de la Carta del 91, a los particulares el ejercicio de funciones administrativas, cuyo alcance y naturaleza jurídica ha generado interesantes controversias, en campos como los de las Cámaras de Comercio y el servicio notarial ${ }^{6}$. También se destacan los artículos. 1 y 82 del Código Contencioso Administrativo de 1984, que hace aplicable ese estatuto a la función administrativa desarrollada por particulares, la Ley 388 de 1997, relativa a las curadurías urbanas, y la Ley 489 de 1998, que regula en forma general el traslado de tales funciones a ese tipo de personas.

6 Sobre estos dos temas véanse algunos comentarios del profesor JAIME VIDAL PERDOMO en el articulo: La colaboración de los particulares en la Actividad Administrativa, publicado en el libro El Derecho Administrativo en Latinoamérica, volumen II, Bogotá, Ediciones Rosaristas, 1986, pá.312 y en su obra Derecho Administrativo, Bogotá: Témis, 1994, p. 69-72. 
Ese nuevo modelo de Estado y la creciente participación de la sociedad en el desarrollo de funciones administrativas, como lo examina la doctrina comparada ${ }^{7}$, se inscribe dentro del triunfo casi universal del neoliberalismo, de la desregulación o neoregulación, así como de la privatización, que se dan en muchos países de todos los continentes y se presenta junto con una nueva concepción del servicio público en sociedades de mercado ${ }^{8}$. Estos hechos contemporáneos íntimamente ligados a la integración y la globalización, generan un nuevo contexto para el Derecho Administrativo, cuyos alcances implican la necesidad de examinar cómo este tipo de ordenamiento jurídico está respondiendo a esas nuevas concepciones y circunstancias y cómo el Estado, en su condición de regulador del ejercicio de la función administrativa, asume el papel que le corresponde.

\section{REFLEXIÓN FINAL}

Una conclusión general puede extraerse de los anteriores comentarios: el mundo actual obedece a unas nuevas circunstancias en el marco de la integración y la globalización, que exigen una mirada igualmente nueva para el Estado y el Derecho Administrativo. En estos campos se están generando cambios que rompen los paradigmas tradicionales y no se puede ignorar la necesidad de adaptar el universo jurídico a los requerimientos de la sociedad actual.

7 ARIÑO ORTIZ, Gaspar. Principios de Derecho Público Económico. Granada: Fundación de Estudios de Regulación y Comaraes, 2000, p. 558

8 MODERNE, FRANCK. Apuntes de Derecho Administrativo Comparado Bogotá: Universidad del Rosario, 2002, p. 27-34. 